\title{
Alimentary Tract Reconstruction following Pancreatoduodenectomy: A New Method with Improvements in the Nutritional State and Gastrointestinal Hormone Release in Dogs
}

\author{
Mikio Imamura, Hidemi Yamauchi and Kenji Kakizaki \\ Department of Surgery, Sendai National Hospital, Sendai \\ 983
}

Imamura, M., Yamauchi, H. and Kakizaki, K. Alimentary Tract Reconstruction following Pancreatoduodenectomy: A New Method with Improvements in the Nutritional State and Gastrointestinal Hormone Release in Dogs. Tohoku J. Exp. Med., 1991, $164(2), 111-123$ — Experiments were performed on adult beagle dogs to investigate the nutritional state and the release of gastrointestinal hormones after pancreatoduodenectomy. A new variation of reconstruction of the alimentary tract was devised and compared with the classical Child's method. In our method, the remaining stomach was anastomosed to the oral stump of the jejunum in an end-to-end fashion. A short mid- intestinal segment was interposed between the pancreatic and bile ducts and the upper jejunum. During the observation period of six months after surgery, the loss of body weight was significantly smaller in the new method, and the frequency of both morbidity and mortality was lower. Although perforated stomal ulcer was observed in one dog after the Child's method, no peptic ulcer was detectable after our method. The integrated increase of plasma triglyceride in response to ingested butter was slightly greater in our method than in the Child's. At six weeks after surgery, plasma concentrations of both cholecystokinin and secretin, not only during fasting but after intake of butter, were augmented in both groups, particularly in the group receiving the new method. These results indicate that retaining the remaining upper small intestine as the food pathway is effective in maintaining good nutritional state, and in facilitating the release of gastrointestinal hormones. - dog; pancreatoduodenectomy ; alimentary tract reconstruction ; cholecystokinin ; secretin

The typical pancreatoduodenectomy procedure involves a massive resection of the digestive organs in the upper abdomen : the resection of the distal stomach, the duodenum, the upper jejunum, the gallbladder and the bile duct together with the head of the pancreas. As a result, a number of undesirable consequences, such as maldigestion of nutrients, glucose intolerance and disturbances in bone metabolism have been brought about (Miyata et al. 1974; Sato et al. 1986; Yagi et al.

Received February 7, 1991; revision accepted for publication May 14, 1991.

Address correspondence to: Mikio Imamura, M.D., Department of Surgery, Sendai National Hospital, 2-8-8 Miyagino, Miyagino-ku, Sendai 983, Japan. 
1986). Therefore, several modifications of reconstruction of the alimentary tract following pancreatoduodenectomy have been divised to decrease the occurrence of postoperative morbidity and mortality. The procedure of Traverso and Longmire (1978), that is the pylorus-preserving pancreatoduodenectomy, was of great interest to us because there is no postgastrectomy syndrome. However, several complications of this procedure have still been reported, including delay in gastric emptying (Braasch et al. 1984 ; Itani et al. 1986) and stomal ulcers (Braasch et al. 1986 ; Itani et al. 1986).

Recently, we reported that the standard Whipple's procedure (Whipple et al. 1935) produces glucose intolerance and decrease in gastrointestinal hormone release (Sato et al. 1986). Based on this observation and also on the study of reconstruction of the biliary tract (Imamura et al. 1982, 1988), we have devised a new method for reconstruction of the alimentary tract following pancreatoduodenectomy, as an alternative to Whipple's classical method.

In this study, to examine the influence of reconstructive methods on postoperative nutritional state and gut hormone release, a comparative study between the new variation and the classical Child's method was done using dogs.

\section{Materials and Methods}

Ten adult (2 to 3-year old) beagle dogs of both sexes, weighing between 9.5 and $12.0 \mathrm{~kg}$, were used for this study. For the control study, after an 18-hr fast $2 \mathrm{~g} / \mathrm{kg}$ of butter was given to each dog and consumed in a few minutes. Blood samples were taken from a peripheral vein via an indwelling catheter, and were drawn into ice-chilled glass tubes containing EDTA.2Na $(1.25 \mathrm{mg} / \mathrm{ml}$ blood) and trasylol $(500 \mathrm{KIU} / \mathrm{ml}$ blood) during fasting and $15,30,60,90,120,150$ and $180 \mathrm{~min}$ after ingestion of butter. Immediately after the experiment, plasma was obtained using a refrigerated $\left(4^{\circ} \mathrm{C}\right)$ centrifugation at $2,800 \mathrm{rpm}$ for 15 min. A part of the plasma was used to measure triglyceride concentration, and the remaining plasma was stored at $-20^{\circ} \mathrm{C}$ until the assay of cholecystokinin (CCK) and secretin concentrations.

After these baseline studies, the dogs were subjected to surgery. Under general anesthesia with Nembutal ${ }^{\circledR}(25 \mathrm{mg} / \mathrm{kg})$, laparotomy was performed through a midline incision, and the pancreatoduodenectomy was performed, namely, the left lobe of the pancreas (approximately $50 \%$ of the whole pancreas) was resected and the accessory pancreatic duct was ligated when the pancreas was dissected from the duodenum as much as possible. The common bile duct was then doubly ligated and cut, and the oral part of the duodenum was resected together with the distal stomach $(60 \%)$. The jejunum was transected at the site 10 $\mathrm{cm}$ anal from Treitz's ligament, and the oral portion was resected together with the duodenum except a small part, $3-4 \mathrm{~cm}$ in length, where the main pancreatic duct opens (Fig. 1). After these procedures, the dogs were randomly divided into two groups, in which the alimentary tract was reconstructed by either the classical Child's method or our new variation.

In our method, the remaining stomach was anastomosed to the oral stump of the jejunum in an end-to-end fashion. A short mid-intestinal segment (15 cm in length) was then constructed and placed beneath the liver retrocolically. To avoid distortion of the interposed segment, the distal stump of the remaining duodenum was closed, and the proximal stump was anastomosed to the mid-intestinal segment in an end-to-end fashion. An end-to-side cholecystoenterostomy was done at the site $5 \mathrm{~cm}$ distal to the duodenoenteros- 


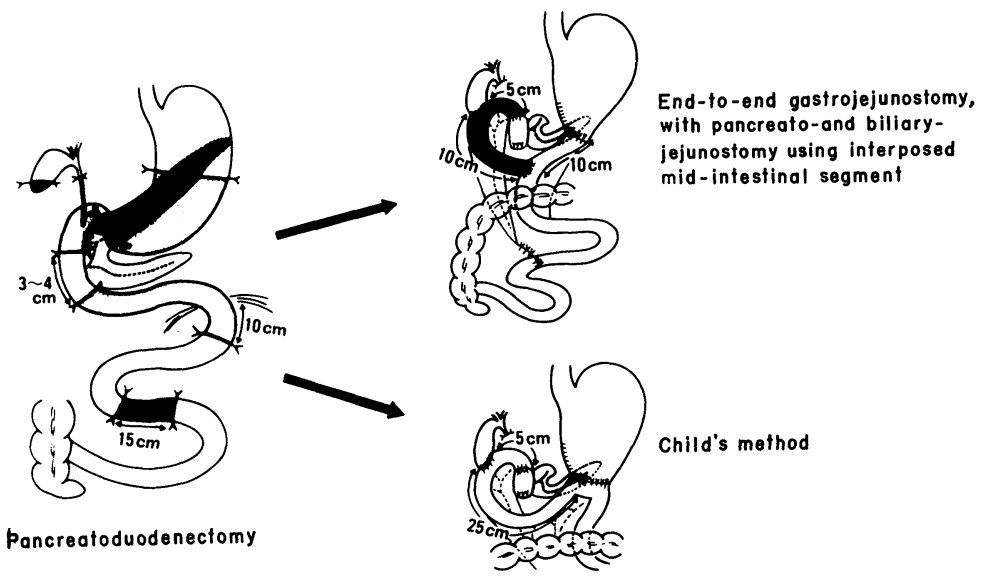

Fig. 1. Two methods of canine alimentary tract reconstruction following pancreatoduodenectomy.

tomy, followed by an end-to-side enteroenterostomy $10 \mathrm{~cm}$ distal to the gastrojejunostomy. On the other hand, in the Child's method, the jejunal stump was placed beneath the liver retrocolically, and anastomosed to the oral stump of the duodenal pouch in an end-to-end fashion, and an end-to-side cholecystojejunostomy at the site $5 \mathrm{~cm}$ distal to the duodenojejunostomy, and an end-to-side gastrojejunostomy at the site $25 \mathrm{~cm}$ distal to the cholecystojejunostomy followed (Fig. 1).

For five days after surgery, each dog was not allowed to drink or eat at all, and took intravenous infusion of $500 \mathrm{ml}$ of the acetate Ringer solution with antibiotics and $100 \mathrm{mg}$ of Gabexate mesilate (FOY ${ }^{\circledR}$, Ono Pharmaceuticals Co., Osaka).

At about 6 weeks after the surgical procedure, experiments were done in the same way as before surgery. To evaluate the nutritional state, body weight was measured every month. Blood biochemical studies were also performed before surgery, and three and six months after surgery. The dogs were observed for six months after surgery, and the experiments were done under appropriate circumstances. After the experiments at six months, dogs were sacrificed after an 18-hr fast, and examined if there was a stomal ulcer or reflux of food into the intestinal segment which had been used for the reconstruction. For dogs which died during the observation period, autopsies were performed and the same investigations were done.

Plasma concentration of CCK was measured by the radioimmunoassay (Himeno et al. 1983) using the antiserum OAL-656, which crossreacts with both CCK- 8 and CCK-33 $(100 \%)$, and with CCK-39 $(84.6 \%)$. Bound hormone was separated from free hormone using the double antibody method. The detection limit was $7.5 \mathrm{pg} / \mathrm{ml}$.

Plasma concentration of secretin was determined by the radioammunoassay method of Chang and Chey (1980), with a modification by Shiratori et al. (1983). A 1-ml sample of plasma was extracted with pure ethanol to remove non-specifically interfering substances and assayed with anti-secretin serum R-1-5. The sensitivity was $1 \mathrm{pg} / \mathrm{ml}$.

Plasma concentration of triglyceride was measured by an enzymatic method using a kit (Unikit Triglyceride- ${ }^{\circledR}$, Chugai Pharmaceuticals Co., Tokyo).

A blood biochemical study was done on fasting plasma concentrations of total protein, albumin, total bilirubin, glutamic-oxaloacetic transaminase (GOT), glutamic-pyruvic transaminase (GPT), alkaline phosphatase (ALP), and calcium.

The results were expressed as mean \pm standard error of the mean (s.E.). The integrated 
responses to butter of triglyceride, CCK and secretin were calculated by the method of Taylor et al. (1978). Student's $t$-test was used to evaluate the statistical significance for paired and unpaired data, and differences with a $p$ value of less than 0.05 were considered statistically significant.

\section{RESULTS}

\section{Postoperative course}

As shown in Table 1, in the group where the modified method was used, three of five dogs survived until six months and were sacrificed after the study of that period. Though two of five dogs died during the observation period, only one dog died from emaciation. On the other hand, in the Child's method group only two of five dogs remained alive up to six months. One dog died of acute diffuse peritonitis due to perforation of a large stomal ulcer at two months after surgery, one from emaciation, and one of volvulus of the large intestine.

\section{Blood biochemical analysis}

Table 2 shows the data of blood biochemical analysis at several time points. In both groups, plasma protein and albumin levels were similar to those before surgery. Jaundice was not observed in either group. Plasma levels of GOT, GPT, and ALP were elevated postoperatively, and they tended to be higher in the Child's method group than in the modified method group. Plasma calcium levels were slightly lower in the former than in the latter.

\section{Body weight}

In both groups, body weight decreased postoperatively as shown in Fig. 2.

TABLE 1. Postoperative course and prognosis

\begin{tabular}{|c|c|c|c|c|}
\hline & \multirow{2}{*}{$\begin{array}{l}\text { Dog } \\
\text { No. }\end{array}$} & \multicolumn{2}{|c|}{ Body weight (kg) } & \multirow{2}{*}{ Cause and time of death } \\
\hline & & Preop. & Postop. & \\
\hline \multirow{5}{*}{$\begin{array}{l}\text { Modified method group } \\
\qquad(n=5)\end{array}$} & 1 & 12 & 11 & Not clear (3 months) \\
\hline & 2 & 12 & 10 & Sacrifice (6 months) \\
\hline & 7 & 10 & 9.5 & Sacrifice (6 months) \\
\hline & 9 & 10 & 7.0 & Emaciation (3 months) \\
\hline & 12 & 10 & 7.5 & Sacrifice (6 months) \\
\hline \multirow{5}{*}{$\begin{array}{l}\text { Child's method group } \\
\qquad(n=5)\end{array}$} & 3 & 9.5 & 7.0 & $\begin{array}{l}\text { Volvulus of the large intestine } \\
\text { (4 months) }\end{array}$ \\
\hline & 5 & 11 & 8.0 & Sacrifice (6 months) \\
\hline & 8 & 10 & 8.0 & Sacrifice (6 months) \\
\hline & 10 & 11 & 8.5 & $\begin{array}{l}\text { Perforation of stomal ulcer } \\
\text { ( } 2 \text { months })\end{array}$ \\
\hline & 11 & 10 & 6.5 & Emaciation (4 months) \\
\hline
\end{tabular}


Alimentary Tract Reconstruction

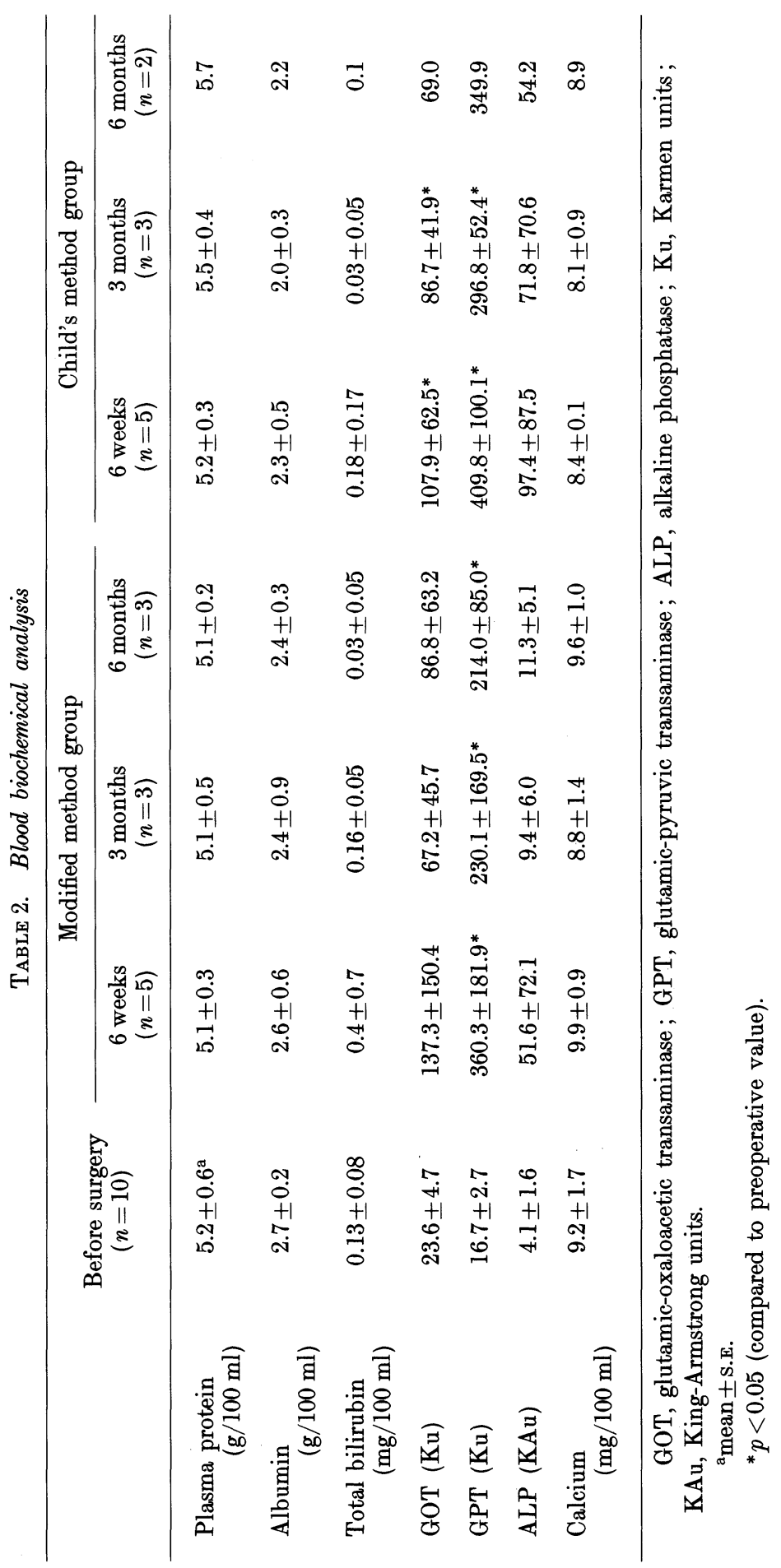




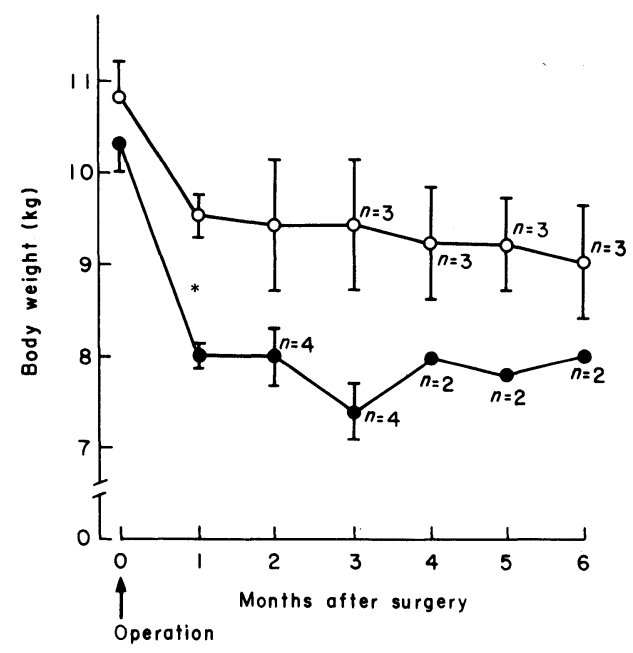

Fig. 2. Changes in body weight following pancreatoduodenectomy. $\bigcirc-O$, modified method $(n=5) ; \bullet-\bullet$ classical Child's method $(n=5)$. Results are expressed as mean \pm S.E. The asterisk represents a significant difference $(p<0.05)$ in comparison between groups.

At one month after surgery, body weight of the Child's method group $(8.0 \pm 0.1 \mathrm{~kg})$ was significantly lower than that of the group on which our method was used $(9.5 \pm 0.2 \mathrm{~kg})$. Compared to each preoperative value, body weight at that time was $88 \pm 2.1 \%$ in the group of our method, and $78 \pm 1.2 \%$ in the Child's method group $(\mathrm{p}<0.05)$. That difference remained almost unchanged during the observation period of six months.

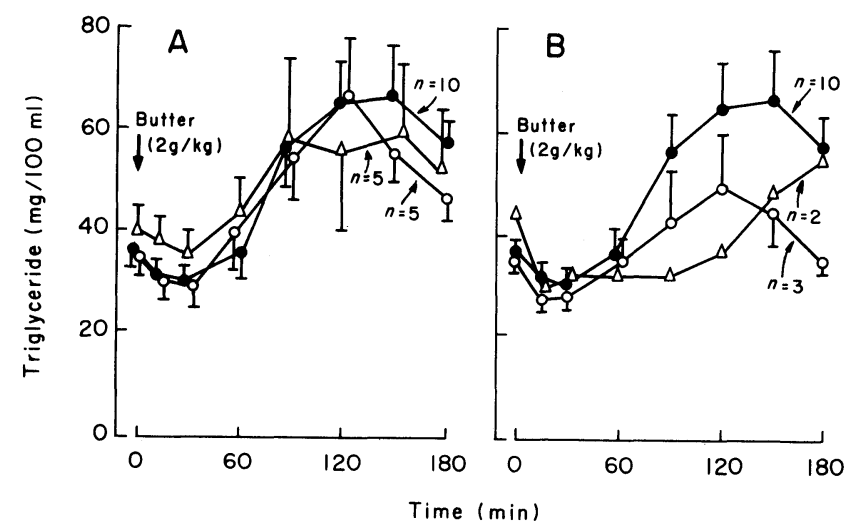

Fig. 3. Changes in plasma triglyceride levels in response to butter. A, 6 weeks after surgery ; B, 6 months after surgery. $\bullet-\bullet$, before surgery $(n=10)$; $\bigcirc-O$, modified method $(n=5) ; \Delta-\Delta$, Child's method $(n=5)$. Values are mean \pm S.E. 
Plasma triglyceride levels in response to butter

At six weeks after surgery, plasma triglyceride concentrations at fasting, in the two operative groups were similar to those before surgery $(35.9 \pm 2.5 \mathrm{mg} / 100$ $\mathrm{ml}$ ) and they increased gradually from one hour after ingestion of butter, reaching the peak levels at around two hours (Fig. 3). The integrated increment of plasma triglyceride was $2.8 \pm 0.6 \mathrm{~g} \cdot 180 \mathrm{~min} / 100 \mathrm{ml}$ before surgery, $2.2 \pm 0.7 \mathrm{~g} \cdot 180 \mathrm{~min} /$ $100 \mathrm{ml}$ in the group of our method, and $1.8 \pm 1.1 \mathrm{~g} \cdot 180 \mathrm{~min} / 100 \mathrm{ml}$ in the Child's method group. Although there were no statistical differences, the value of our method group was closer to the preoperative value than that of the Child's method group.

At six months after surgery, though three dogs were alive in our method group, and only two in the Child's method group, fasting plasma triglyceride levels in these two groups were still similar to those before surgery. After ingestion of butter, however, plasma triglyceride levels were markedly lowered in both operative groups. The response pattern of plasma triglyceride to butter was similar in the preoperative study and our method group, but in the Child's method group the increase of plasma triglyceride levels was markedly delayed.

\section{Plasma CCK concentrations in response to butter}

Fig. 4 shows plasma CCK levels in response to oral ingestion of butter. In the preoperative study, plasma CCK concentration was $15.2 \pm 1.6 \mathrm{pg} / \mathrm{ml}$ during fasting, reaching the peak value $(21.9 \pm 2.7 \mathrm{pg} / \mathrm{ml})$ at $60 \mathrm{~min}$ after feeding, and remained unchanged up to three hours. At six weeks after surgery, fasting plasma CCK concentrations in the two groups $(36.1 \pm 1.2 \mathrm{pg} / \mathrm{ml}$ in the group of our method, and $34.8 \pm 11.1 \mathrm{pg} / \mathrm{ml}$ in the Child's method group) were significantly

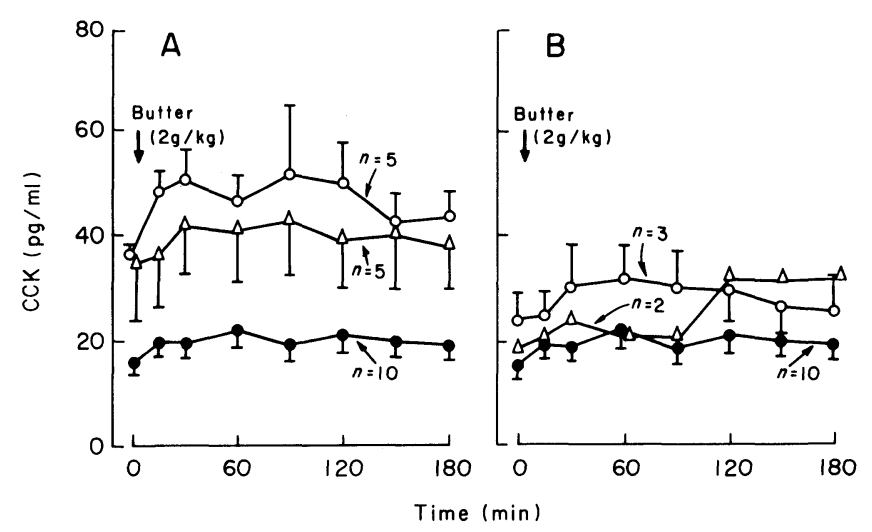

Fig. 4. Changes in plasma immunoreactive cholecystokinin (CCK) levels in response to butter.

A, 6 weeks after surgery ; B, 6 months after surgery. Symbols are as indicated in Fig. 3. 
higher than those before surgery. After ingestion of butter, plasma CCK concentrations increased in the two operative groups, and they showed higher levels than those in the preoperative study, especially in the modified method group. Although they failed to reach statistical significance, the integrated incremental responses of CCK to butter in both groups $(1.92 \pm 1.13 \mathrm{ng} \cdot 180 \mathrm{~min} / \mathrm{ml}$ in the group of our method, and $1.03 \pm 0.74 \mathrm{ng} \cdot 180 \mathrm{~min} / \mathrm{ml}$ in the Child's method group) were higher than that before surgery $(0.85 \pm 0.26 \mathrm{ng} \cdot 180 \mathrm{~min} / \mathrm{ml})$. Comparing the two operative groups, the response was slightly higher in our method group than in the Child's method.

At six months after surgery, in both groups plasma CCK concentrations approached preoperative levels both at fasting and after ingestion of butter, but they were still augmented in the modified method group. In the Child's method group, there was a marked delay in the increase of CCK levels after feeding as in the case of triglyceride.

Plasma secretin concentrations in response to butter

At six weeks after surgery, as shown in Fig. 5, plasma secretin concentrations during fasting were significantly higher in both operative groups $(3.2 . \pm 0.4 \mathrm{pg} / \mathrm{ml}$ in the modified method group, and $3.8 \pm 0.7 \mathrm{pg} / \mathrm{ml}$ in the Child's method group) than those before surgery $(1.7 \pm 0.4 \mathrm{pg} / \mathrm{ml})$. After ingestion of butter, plasma secretin concentrations increased significantly in the two groups as in the preoperative study. The peak secretin levels in both groups $(8.5 \pm 2.3 \mathrm{pg} / \mathrm{ml}$ in the modified method group, and $7.5 \pm 1.0 \mathrm{pg} / \mathrm{ml}$ in the Child's method group) were slightly higher than those before surgery $(7.0 \pm 1.0 \mathrm{pg} / \mathrm{ml})$. The integrated incremental responses of secretin to butter were $444.2 \pm 73.1 \mathrm{pg} \cdot 180 \mathrm{~min} / \mathrm{ml}$ in the preoperative study, $627.9 \pm 130.3 \mathrm{pg} \cdot 180 \mathrm{~min} / \mathrm{ml}$ in the modified method group,

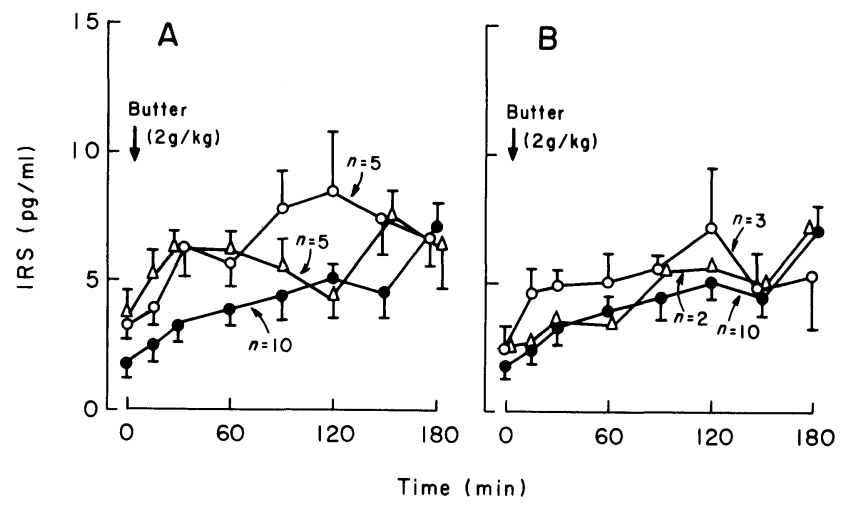

Fig. 5. Changes in plasma immunoreactive secretin (IRS) levels in response to butter.

A, 6 weeks after surgery ; B, 6 months after surgery. Symbols are as indicated in Fig. 3. 
and $371.3 \pm 125.6 \mathrm{pg} \cdot 180 \mathrm{~min} / \mathrm{ml}$ in the Child's method group. Although they could not reach a significant difference, the response in the group of our method was higher than those in both the preoperative study and the Child's method group.

At six months after surgery, as in the case of CCK, in each group plasma secretin levels came close to preoperative levels, but they were still slightly higher in the modified method group.

\section{Discussion}

Postoperative complications of pancreatoduodenectomy seem to be closely related to the reconstruction method of the alimentary tract as well as the extensive surgery. Accordingly, a number of modifications of the classical Whipple's procedure (Whipple et al. 1935) have been devised by many surgeons. In 1960, Imanaga devised a new reconstruction method where the remaining stomach was anastomosed to the upper jejunum in the Billroth-I type to preserve the function of the liver and pancreas, and both the bile and pancreatic ducts were anastomosed directly to the upper jejunum in an end-to-side fashion. Yamashita et al. (1981) interposed the uppermost part of the remaining jejunum, $40 \mathrm{~cm}$ in length, between the remaining stomach and the Roux-en-Y loop of the jejunum, and reported that the pancreatic exocrine secretion was preserved well. After Traverso and Longmire (1978) devised the pylorus-preserving procedure, several modifications have been done on the method (Flautner et al. 1985 ; Kim et al. 1987 ; Takada et al. 1989). Kim et al. (1987) anastomosed the duodenal bulb to the stump of the jejunum as the method of Watson (1944), and both the bile and pancreatic ducts to the upper jejunum as the method of Imanaga (1960). Takada et al. (1989) also did the reconstruction in the Billroth-I type after the pyloruspreserving pancreato-duodenectomy, but the pancreatic duct was anastomosed to the stomach as the method of Flautner et al. (1985). In contrast to the classical Whipple's procedure, in the pylorus-preserving pancreatoduodenectomy the function of the stomach, such as reserving, mixing and finally breaking down food, can be preserved, and bring about better nutritional status (Suzuki et al. 1988). Despite these merits, there are some serious complications. The delay of gastric emptying has been observed frequently (Braasch et al. 1984 ; Itani et al. 1986). The occurrence of stomal ulceration has also been reported, and vagotomy with antrectomy was added afterwards in some cases (Itani et al. 1986).

In our previous studies of the reconstruction methods of the biliary tract (Imamura et al. 1982), jejunal interposition hepaticoduodenostomy with a short jejunal segment was evaluated to be a more physiologic procedure, approximating the organ more closely to the original state, from the viewpoints of gastric acid secretion and the release of gastrointestinal hormones, than Roux-en-Y hepaticojejunostomy or the interposition method with a long jejunal segment. As mentioned above, our previous study of patients undergoing pan- 
creatoduodenectomy (Sato et al. 1986) suggested that the diminished release of some gastrointestinal hormones resulted from the diversion of the stream of food away from proximal portion of the small intestine as well as the massive resection of digestive organs.

From these experiences, we have devised a variation of the alimentary tract reconstruction after pancreatoduodenectomy as shown in Fig. 1. It was intended that our method would have the following characteristics. Firstly, the gastrojejunostomy was the Billroth-I type, to allow maximum utilization of the upper small intestine for effective digestion and absorption of nutrients, and for efficient release of gastrointestinal hormones. Secondly, the extent of gastric resection was made smaller than usual to preserve the reservoir function of the stomach as much as possible. Thirdly, a short segment of the small intestine was interposed between the food stream and the pancreatic and bile ducts to prevent retrograde cholangitis and obstruction of the pancreatic duct. Fourthly, a mid-intestinal segment was used for the reconstruction, so as not to impair digestion and absorption of nutrients and the release of gut hormones. Finally, both pancreatic juice and bile were allowed to flow into the upper jejunum to contact food and gastric acid effectively.

In the classical Child's method, the most proximal part of the jejunum, about $40 \mathrm{~cm}$ in length, was used as a kind of blind loop to let both pancreatic juice and bile flow. As a result, it can be easily speculated that the release of certain gastrointestinal hormones such as secretin, CCK and gastric inhibitory polypeptide, which play important roles in the suppression of gastric acid secretion, stimulation of pancreatic exocrine secretion and enteroinsular axis (Dupre et al. 1973 ; Faichney et al. 1981; You and Chey 1987; Watanabe et al. 1988), will be reduced after surgery. Therefore, it seems ideal not to exclude the remaining upper jejunum from the stream of food. The ileum is another important part for the release of some gut hormones such as peptide YY (PYY), neurotensin and enteroglucagon (Ghatei and Bloom 1981; Aponte et al. 1985 ; Doyle et al. 1985), and also for the enterohepatic circulation of bile (Dietschy 1968). These are reasons why we used the mid-portion of the small intestine for the pathway of bile and pancreatic juice. A short length of the interposed segment is deemed better not only to prevent stasis of both digestive juice and food, but also to preserve the effective release of gut hormones (Imamura et al. 1982).

In the present study, postoperative decrease of body weight was smaller in our new variation than in the classical Child's method, the integrated increase of plasma triglyceride in response to butter ingestion and plasma levels of CCK and secretin were higher in the former. Moreover, stomal ulcer was detectable only in one dog undergoing the Child's method. These results can well satisfy our intention which was mentioned previously.

The negative feedback regulation mediated by CCK between tryptic activity in the upper small intestine and pancreatic exocrine secretion has been shown in 
rats (Louie et al. 1986) and humans (Owyang et al. 1986). In this study, plasma CCK levels both during fasting and after intake of butter were higher after pancreatoduodenectomy compared to preoperative levels. Therefore, it is speculated that the negative feedback regulation may function after the pancreatoduodenectomy where about $50 \%$ of the pancreas was resected. Comparing the two kinds of reconstruction methods, plasma CCK concentration tended to be higher in our method than in the Child's method. It is considered that, in our modification, the most proximal part of the remaining jejunum can more effectively contact with food which is digested by bile and pancreatic juice, resulting in more efficient stimulation of CCK-releasing cells (Watanabe et al. 1988). Regarding higher levels of plasma secretin after surgery than preoperative levels, which were especially evident in the new variation, similar speculation may be possible. In this respect, Sun et al. (1986) and Shiratori et al. (1989) have reported that there exists the negative feedback mechanism between the activity of pancreatic enzymes in the duodenum and pancreatic exocrine secretion in rats and dogs, which is mediated by secretin. Plasma concentrations of both CCK and secretin approached preoperative levels with time after surgery. This may suggest that the exocrine function of the remaining pancreas has been recovering with time.

Secretin is one of the candidates of enterogastrone (You and Chey 1987). Therefore, it seems to be very favorable to keep plasma secretin levels higher for suppressing gastric acid secretion and preventing the occurrence of peptic ulceration after pancreatoduodenectomy. This also indicates that the extent of gastric resection can be made less in our method, and the function of the stomach may be preserved well.

In conclusion, it is considered that retaining the remaining upper small intestine as the main food pathway is most effective for maintaining good nutritional state and facilitating the release of certain gastrointestinal hormones such as CCK and secretin. One other point suggested by this study is that negative feedback regulation between intraluminal activity of pancreatic enzymes in the upper small intestine and pancreatic exocrine secretion may function through the mediation of $\mathrm{CCK}$ and/or secretin even after pancreatoduodenectomy. It is also expected that the patient's nutritional state can be improved markedly by using our variation for reconstruction of the alimentary tract following pancreatoduodenectomy.

\section{Acknowledgments}

We express our appreciation to Drs. K. Shiratori, S. Watanabe and T. Takeuchi (Department of Gastroenterology, Tokyo Women's Medical College, Tokyo, Japan) for the measurement of plasma secretin concentration. 


\section{References}

1) Aponte, G.W., Fink, A.S., Meyer, J.H., Tatemoto, K. \& Taylor, I.L. (1985) Regional distribution and release of peptide YY with fatty acids of different chain length. $A m$. J. Physiol., 249, G 745-750.

2) Braasch, J.W., Gongliang, J. \& Rossi, R.L. (1984) Pancreatoduodenectomy with preservation of the pylorus. World J. Surg., 8, 900-905.

3) Braasch, J.W., Rossi, R.L., Watkins, E., Jr., Deziel, D.J. \& Winter, P.F. (1986) Pyloric and gastric preserving pancreatic resection. Experience with 87 patients. Ann. Surg., 204, 411-418.

4) Chang, T.-M. \& Chey, W.Y. (1980) Radioimmunoassay of secretin : A critical review and current status. Dig. Dis. Sci., 25, 529-552.

5) Dietschy, J.M. (1968) Mechanisms for the intestinal absorption of bile acids. J. Lipid Res., 9, 297-309.

6) Doyle, H., Greeley, G.H., Jr., Mate, L., Sakamoto, T., Townsend, C.M., Jr. \& Thompson, J.C. (1985) Distribution of neurotensin in the canine gastrointestinal tract. Surgery, 97, 337-341.

7) Dupre, J., Ross, S.A., Watson, D. \& Brown, J.C. (1973) Stimulation of insulin secretion by gastric inhibitory polypeptide in man. J. Clin. Endocrinol. Metab., 37, 826-828.

8) Faichney, A., Chey, W.Y., Kim, Y.C., Lee, K.Y., Kim, M.S. \& Chang, T.-M. (1981) Effect of sodium oleate on plasma secretin concentration and pancreatic secretion in dog. Gastroenterology, 81, 458-462.

9) Flautner, L., Tihanyl, T. \& Szécsény, A. (1985) Pancreatogastrostomy : An ideal complement to pancreatic head resection with preservation of the pylorus in the treatment of chronic pancreatitis. Am. J. Surg., 150, 608-611.

10) Ghatei, M.A. \& Bloom, S.R. (1981) Enteroglucagon in man. In: Gut Hormones, edited by S.R. Bloom \& J.M. Polak. Churchill Livingstone, London-New York, pp. $332-338$.

11) Himeno, S., Tarui, S., Kanayama, T., Kurokawa, M., Shinomura, Y., Hayashi, C., Tateischi, K., Imagawa, K., Hashimura, E. \& Hamaoka, T. (1983) Plasma cholecystokinin responses after ingestion of liquid meal and intraduodenal infusion of fat, amino acids, or hydrochloric acid in man: Analysis with region specific radioimmunoassay. Am. J. Gastroenterol., 78, 703-707.

12) Imanaga, H. (1960) A new method of pancreaticoduodenectomy designed to preserve liver and pancreatic function. Surgery, 47, 577-586.

13) Imamura, M., Kameyama, J., Ohneda, A. \& Sato, T. (1982) Effect of various reconstructions of the biliary tract upon the secretion of gastric acid and gastrointestinal hormones in dogs. Surg. Gastroenterol., 1, 105-114.

14) Imamura, M., Takahashi, M., Sasaki, I., Yamauchi, H. \& Sato, T. (1988) Effects of the pathway of bile flow on the digestion of fat and the release of gastrointestinal hormones. Am. J. Gastroenterol., 83, 386-392.

15) Itani, K.M.F., Coleman, R.E., Akwari, O.E. \& Meyers, W.C. (1986) Pyloruspreserving pancreatoduodenectomy: A clinical and physiologic appraisal. Ann. Surg., 204, 655-664.

16) Kim, H-C., Suzuki, T., Kajiwara, T., Miyashita, T., Imamura, M. \& Tobe, T. (1987) Exocrine and endocrine stomach after gastrobulbar preserving pancreatoduodenectomy. Ann. Surg., 206, 717-727.

17) Louie, D.S., May, D., Miller, P. \& Owyang, C. (1986) Cholecystokinin mediates feedback regulation of pancreatic enzyme secretion in rats. Am. J. Physiol., 250, G 252-259.

18) Miyata, M., Nakao, T., Uozumi, T., Okamoto, E. \& Manabe, H. (1974) Insulin 
secretion after pancreatoduodenectomy. Ann. Surg., 179, 494-498.

19) Owyang, C., Louie, D.S. \& Tatum, D. (1986) Feedback regulation of pancreatic enzyme secretion-suppression of cholecystokinin release of trypsin. J. Clin. Invest., 77, 2042-2047.

20) Sato, T., Imamura, M., Matsuno, S., Sasaki, I. \& Ohneda, A. (1986) Gastric acid secretion and gut hormone release in patients undergoing pancreaticoduodenectomy. Surgery, 99, 728-734.

21) Shiratori, K., Watanabe, S. \& Takeuchi, T. (1983) Radioimmunoassay of secretin with special reference to examination of its sensitivity. Nippon Shokakibyo Gakkai Zasshi, 80, 1475-1479. (in Japanese with English abstract)

22) Shiratori, K., Jo, Y.H., Lee, K.Y., Chang, T.-M. \& Chey, W.Y. (1989) Effect of pancreatic juice and trypsin on oleic acid-stimulated pancreatic secretion and plasma secretin in dogs. Gastroenterology, 96, 1330-1336.

23) Sun, G., Lee, K.Y., Chang, T.-M. \& Chey, W.Y. (1986) Role of endogenous secretin in negative feedback regulation of exocrine pancreas in rats. Dig. Dis. Sci., 31, 29. (Abstract)

24) Suzuki, T., Imamura, M., Kajiwara, T., Kim, H.-C., Miyashita, T. \& Tobe, T. (1988) A new method of reconstruction after pylorus- preserving pancreatoduodenectomy. World J. Surg., 12, 645-650.

25) Takada, T., Yasuda, H., Shikata, J., Watanabe, S., Shiratori, K. \& Takeuchi, T. (1989) Postprandial plasma gastrin and secretin concentrations after a pancreatoduodenectomy: A comparison between a pylorus-preserving pancreatoduodenectomy and the Whipple procedure. Ann. Surg., 210, 47-51.

26) Taylor, I.L., Feldman, M., Richardson, C.T. \& Walsh, J.H. (1978) Gastric and cephalic stimulation of human pancreatic polypeptide release. Gastroenterology, $\mathbf{7 5}$, 432-437.

27) Traverso, L.W. \& Longmire, W.P., Jr. (1978) Preservation of the pylorus in pancreaticoduodenectomy. Surg. Gynecol. Obstet., 146, 959-962.

28) Watanabe, S., Lee, K.Y., Chang, T.-M., Berger-Ornstein, L. \& Chey, W.Y. (1988) Role of pancreatic enzymes on release of cholecystokinin-pancreozymin in response to fat. Am. J. Physiol., 254, G 837-842.

29) Watson, K. (1944) Carcinoma of ampulla of Vater: Successful radical resection. Br. J. Surg., 31, 368-373.

30) Whipple, A.O., Parsons, W.B. \& Mullians, C.R. (1935) Treatment of carcinoma of the ampulla of Vater. Ann. Surg., 102, 763-779.

31) Yagi, M., Takano, N., Yamaguchi, A., Takeshita, Y., Konishi, K., Fujita, H., Nagakawa, T. \& Miyazaki, I. (1986) A study on radiological assessment of bone density by microdensitometer after pancreatoduodenectomy. Nippon Shokakigeka Gakkai Zasshi., 19, 938-941. (in Japanese)

32) Yamashita, Y., Kasahara, K., Tenmoku, S., Miyata, M. \& Morioka, Y. (1981) Procedures of gastrointestinal reconstruction after pancreatico-duodenectomy and exocrine pancreatic function of the remnant pancreas. Nippon Shokakigeka Gakkai Zasshi, 14, 1594-1601. (in Japanese)

33) You, C.H. \& Chey, W.Y. (1987) Secretin is an enterogastrone in humans. Dig. Dis. Sci., 32, 466-471. 